\title{
Antidepressant Actions of Ketamine Mediated by the Mechanistic Target of Rapamycin, Nitric Oxide, and Rheb
}

\author{
Maged M. Harraz ${ }^{1}$ Solomon H. Snyder ${ }^{1,2,3}$
}

Published online: 13 June 2017

(C) The American Society for Experimental NeuroTherapeutics, Inc. 2017

\begin{abstract}
The weeks/months it takes for traditional antidepressants to act pose an obstacle in the management of depression. Ketamine's prompt and sustained antidepressant effects constitute a major advance. Multiple studies implicate glutamatergic signaling to protein synthesis machinery and synapse formation in ketamine's antidepressant effects. Here we review evidence linking ketamine to glutamate receptor subtypes and protein homeostasis. We describe a signaling cascade wherein nitric oxide drives the formation of a ternary protein complex comprised of glyceraldehyde 3-phosphate dehydrogenase, seven in absentia homolog 1 , and Ras homo$\log$ enriched in brain downstream of the glutamate $\mathrm{N}$-methylD-aspartate receptor. Seven in absentia homolog 1 ubiquitylates and degrades Ras homolog enriched in brain leading to inhibition of mechanistic target of rapamycin. Ketamine inhibits this molecular cascade leading to activation of mechanistic target of rapamycin and, in turn, to antidepressant actions.
\end{abstract}

Keywords Ketamine $\cdot$ Nitric oxide $\cdot$ Glutamate receptor . GAPDH $\cdot$ Rheb $\cdot$ mTOR

Solomon H. Snyder

ssnyder@jhmi.edu

1 The Solomon H. Snyder Department of Neuroscience, Johns Hopkins University School of Medicine, Baltimore, MD 21205, USA

2 Department of Psychiatry and Behavioral Sciences, Johns Hopkins University School of Medicine, Baltimore, MD 21205, USA

3 Department of Pharmacology and Molecular Sciences, Johns Hopkins University School of Medicine, Baltimore, MD 21205, USA

\section{Introduction}

The excitatory neurotransmitter glutamate acts through several subtypes of receptors of which the $N$-methyl-D-aspartate (NMDA) and $\alpha$-amino-3-hydroxy-5-methyl-4isoxazolepropionic acid (AMPA) [1] receptors (AMPARs) have been most investigated. Roles for NMDA neurotransmission in psychiatry have been proposed for both schizophrenia and affective disorders. One key advance was the appreciation that the psychotomimetic actions of the widely abused drug phencyclidine (PCP; angel dust) reflect blockade of NMDA receptors [2]. At about this time there was increasing evidence that excessive NMDA neurotransmission mediates neurotoxicity and brain damage associated with vascular strokes [3]. Accordingly, a number of major pharmaceutical concerns developed NMDA antagonists as possible antistroke drugs. One of these, MK801, was potent and selective but then shown to elicit psychotomimetic actions. This led to an interest in glutamate-NMDA agonists for the possible treatment of schizophrenia. Glutamate itself lacks appropriate bioavailability for oral administration in humans and access across the blood-brain barrier. Appreciation that the Disomer of serine is a co-agonist with glutamate at NMDA receptors led to studies characterizing its behavioral effects. A number of investigations have indicated beneficial effects of D-serine and D-cycloserine in schizophrenic patient populations [4].

Based on these considerations Krystal et al. [5] initiated studies of ketamine in human subjects. The initial investigations employed intravenous doses of ketamine. As ketamine is an anesthetic agent, care was taken to employ subanesthetic doses. At these low doses patient mood was enhanced, and detailed analysis revealed a clinically relevant antidepressant action [6]. The antidepressant effects commence within a few hours of drug administration and often last a week or more 
after a single administration. Ketamine improves a number of core depressive symptoms including anhedonia [7], suicidal thoughts [8], and depressed mood [9]. It is active both in unipolar and bipolar depressed patients who have proved refractory to diverse drug therapy regimens. Antidepressant actions of ketamine were subsequently demonstrated in multiple animal models of depression, including the forced swimming test, a learned helplessness model, novelty-suppressed feeding, chronic unpredictable stress paradigm, and chronic social defeat stress and social interaction [10-12].

Selectivity of the ketamine effect for NMDA receptors has been explored by studies of other NMDA antagonists. In rodent models of depression NMDA antagonists, including MK-801, 2-amino-7-phosphonoheptanoic acid, and Ro 256981 , have proved efficacious $[10,11,13]$. NMDA antagonists such as CP-101,606 have elicited ketamine-like beneficial actions in depressed patients [14]. However, there have been some discrepancies. For instance, in a study of 8 patients with major depressive disorder, Ferguson et al. [15] demonstrated early efficacy of memantine, a selective NMDA antagonist. However, other studies found memantine ineffective in patients with major depression [16, 17]. One potential explanation for the discrepancy between these studies involves the administered dose of memantine. Thus, ketamine and memantine displayed comparable occupancy at MK-801/ NMDA receptor sites when administered at the same dose. However, ketamine had much faster kinetics with rapid binding followed by swifter dissociation than memantine [18]. Administration of memantine at 20 to $40 \mathrm{mg}$ daily alleviated depression symptoms [15, 19]. However, lower doses of memantine, 5 to $20 \mathrm{mg}$ daily, resulted in lack of favorable effect in patients with depression $[16,17]$. Ketamine, however was consistently used in a dose comparable to the higher memantine dose, $0.5 \mathrm{mg} / \mathrm{kg}$ of body weight, in several studies $[6,8,9,20]$. Nonetheless, it is not clear whether the different doses of memantine caused the various clinical outcomes. Gideons et al. [21] demonstrated antidepressant actions in mice for ketamine but not memantine. While both ketamine and memantine block the NMDA receptor at rest in the absence of magnesium, ketamine but not memantine blocks the NMDA receptor in the presence of physiologic levels of magnesium. Furthermore, memantine failed to inhibit the phosphorylation of eEF2 or upregulate brain-derived neurotrophic factor (BDNF) protein expression, which is crucial for ketamine's antidepressant-like effect [21]. For a detailed discussion, Johnson et al. [22] recently reviewed potential explanations for discrepancies in the clinical effects of ketamine and memantine in patients with major depression.

The pathways downstream of NMDA receptors may be differentially affected by diverse NMDA antagonists. For instance, BDNF is associated with mood enhancement and has been implicated in the actions of various antidepressant drugs. PCP, a noncompetitive NMDA receptor antagonist, caused a transient increase in intracellular BDNF but inhibited its secretion in cortical cultures. PCP-mediated reduction of BDNF secretion inhibited tyrosine receptor kinase $\mathrm{B}$ signaling and decreased synaptic connectivity [23]. Xu et al. [24] showed that PCP inhibits BDNF protein levels both in cortical cultures and in mouse frontal cortex. However, ketamine rapidly increased BDNF protein levels in rat hippocampus [25]. Gideons et al. [26] provided evidence that ketamine's antidepressant effects in rodent models reflect enhanced synthesis of BDNF via inactivating eukaryotic elongation factor 2 (eEF2), also known as calmodulin-dependent protein kinase (CaMK) III. Inhibitors of eEF2 kinase elicit rapid antidepressant-like effects.

In a study by Nosyreva et al. [27] ketamine failed to induce an antidepressant effect and synaptic potentiation in juvenile animals. This work implicates developmental factors in ketamine's antidepressant actions. Furthermore, the authors showed that in mature hippocampal slices, ketamine and the NMDA inhibitor D-AP5 induced potentiation of AMPARmediated evoked neurotransmission. These findings are in line with other evidence implicating the AMPAR in ketamine's antidepressant effects [27]. It seems that protein synthesis plays an important role in ketamine's antidepressant effect. For example, stress-induced decrease in synaptic protein levels was reversed by ketamine in a rapamycindependent manner [10, 11]. Furthermore, the protein synthesis inhibitor anisomycin blocked the antidepressant-like effects of ketamine [26].

There is evidence that ketamine may also act by augmenting glutamate-AMPAR-mediated neurotransmission. Thus, Maeng et al. [28] showed that Ro25-6981, an NMDA antagonist selective for the NR2B receptor subtype, elicited antidepressant actions comparable with those of ketamine. Surprisingly, these actions were blocked by the AMPA antagonist NBQX, consistent with mediation of ketamine influences via AMPA systems. Notably compelling is an extensive study by Zanos et al. [12]. These investigators focused upon the roles of ketamine metabolites, as well as ketamine enantiomers, and employed a variety of behavioral models in mice, including forced swimming test, novelty-suppressed feeding test, and learned helplessness. Their findings were not consistent with an NMDA antagonism model. Thus, S-ketamine is about 4 times more potent as an NMDA antagonist than Rketamine, whereas R-ketamine was more potent in several antidepressant behavioral models. The study focused, in particular, upon 2R,6R-hydroxynorketamine (2R,6R-HNK), the major metabolite of ketamine in blood and brain of mice, which appeared to be relatively inactive as an NMDA antagonist but which increased AMPA neurotransmission. 2R,6RHNK exerted robust antidepressant actions and lacked the behavioral adverse effects of ketamine such as motor incoordination. The authors showed decreased eEF2K phosphorylation and increased BDNF protein levels in CD-1 mice 
hippocampus 1 and $24 \mathrm{~h}$ after 2R,6R-HNK injection. However, they did not detect mTOR activation at these 2 time points. Paul et al. [29] showed that $2 \mathrm{~S}, 6 \mathrm{~S}-\mathrm{HNK}$ induces mTOR and p70S6K phosphorylation within $20 \mathrm{~min}$ in rat cerebral cortex [29]. However, experiments of Zanos et al. [12] in CD-1 mice did not show antidepressant-like effects following $2 \mathrm{~S}, 6 \mathrm{~S}-\mathrm{HNK}$ administration. These findings might argue against a role for mTOR in the $2 \mathrm{R}, 6 \mathrm{R}-\mathrm{HNK}$ antidepressant-like effect. It will be interesting to investigate a time course for mTOR activation and test whether blocking mTOR activity regulates $2 \mathrm{R}, 6 \mathrm{R}-\mathrm{HNK}$ induced antidepressant-like effects. Whereas ketamine is an abusable agent, being readily self-administered, no self-administration was evident with 2R,6R-HNK. Thus, besides being a potentially promising therapeutic agent, actions of $2 \mathrm{R}, 6 \mathrm{R}-\mathrm{HNK}$ imply that enhancement of AMPA signaling rather than NMDA antagonism underlies the therapeutic actions of ketamine in depression. However, the identity of the receptor that mediates 2R,6R-HNK antidepressant-like effect remains elusive.

Li et al. [10] demonstrated that ketamine increases synaptic protein synthesis and synaptogenesis in rat prefrontal cortex (PFC) [10]. Rapamycin, a mechanistic target of rapamycin (mTOR) inhibitor, prevented these changes and blocked ketamine's antidepressant effect. In these studies, ketamine increased mTOR activation in rat PFC within 30 min of intraperitoneal injection. Inhibitors of AMPAR, extracellular signal-regulated kinase and phosphatidylinositide 3-kinase blocked ketamine's effect on mTOR activity in rat PFC. A selective NMDA receptor subtype 2B (NR2B) antagonist, Ro 25-6981, mimicked the molecular and behavioral effects of ketamine. Ro 25-6981 activated mTOR, increased synaptic protein synthesis, and resulted in a rapamycin-sensitive antidepressant-like phenotype in rats [10]. Ketamine and Ro 256981 rescued anhedonic and anxiogenic behaviors in rats exposed to 21 days of chronic unexpected stress (CUS). CUS was associated with a decrease in synaptic protein expression and decreased synaptogenesis in rat PFC. Ketamine rapidly reversed the biochemical and structural changes induced by CUS, while rapamycin abolished ketamine's effects [11]. These findings suggest that ketamine's antidepressant action is mediated through mTOR-dependent protein synthesis and downstream synapse formation in an AMPAR-dependent manner. These findings also indicate that NMDA receptor inhibition mimics ketamine's effects on protein synthesis and its antidepressant-like phenotype.

In another study, carried out by Miller et al. [30], selective deletion of NR2B in neocortical glutamatergic principal neurons (2B $\Delta \mathrm{Ctx}$ mice) resulted in an antidepressant-like phenotype in mice. The authors bred an NR2B floxed mouse generated by Brigman et al. [31] with a mouse expressing Cre recombinase enzyme under the glutamatergic neuron-specific NEX promoter generated by Klaus-Armin Nave and his team [32]. 2B $\Delta \mathrm{Ctx}$ mice showed decreased immobility in a tail suspension test, as well as decreased time spent in closed arm and increased time spent in open arm of an elevated plus maze. While ketamine decreased the immobility time of control mice in the tail suspension test, it failed to reduce the immobility time in $2 \mathrm{~B} \Delta \mathrm{Ctx}$ mice. In an open-field test, $2 \mathrm{~B} \Delta \mathrm{Ctx}$ mice showed hyperlocomotion; however, ketamine reduced the distance traveled by both control and $2 \mathrm{~B} \Delta \mathrm{Ctx}$ mice in the open-field test. The investigators also examined the effects of NR2B deletion in glutamatergic neurons on synaptogenesis and synaptic protein expression in the PFC. Similar to ketamine's effects, $2 \mathrm{~B} \Delta \mathrm{Ctx}$ mice had increased miniature excitatory postsynaptic currents in PFC fresh tissue slices compared with control mice. Systemic ketamine administration $24 \mathrm{~h}$ before measurements increased miniature excitatory postsynaptic currents in control mice but not in fresh PFC slices from $2 \mathrm{~B} \Delta \mathrm{Ctx}$ mice. NR2B deletion in glutamatergic neurons elicited alterations resembling those of ketamine injection in control mice, namely increased expression of a number of synaptic proteins and increased activation of mTOR relative to vehicleinjected control mice. Ketamine injection in $2 \mathrm{~B} \Delta \mathrm{Ctx}$ mice failed to alter the expression levels of these synaptic proteins and did not change the activation of mTOR [30]. These findings implicate NR2B in the regulation of mTOR activity, synaptic protein expression levels, glutamatergic synaptogenesis, and antidepressant-like effects. The absence of ketamine effects in $2 \mathrm{~B} \Delta \mathrm{Ctx}$ mice is consistent with a role for NR2B in ketamine's antidepressant actions. In support of these finding, Engelhardt et al. [33] used Black/White Alley, Light-Dark Box, and modified elevated plus maze paradigms to demonstrate similar diminution of the anxious phenotype in mice in which NR2B was deleted postnatally under the $\mathrm{Ca}^{2+} / \mathrm{CaMKII}$ promoter [33] . However, using the same genetic manipulation, Andrew Holmes and his team observed a weaker phenotype in CaMKII driven Cre recombinase deletion of NR2B in mice. These NR2B conditional knockout (CKO) mice did not differ from controls in anxious behavior or in a 1-session forced swim test. However, these NR2B CKO mice had reduced immobility compared with controls in a repeated inescapable forced swim test [34]. These findings differed from those in other NR2B CKO studies. One explanation might lie in the difference between the brain subregions with successful NR2B deletion. The NEX promoter used by Miller et al. [30] to drive Cre recombinase produced the most widespread NR2B gene deletion among the 3 studies. Engelhardt et al. [33] reported a distribution of NR2B gene deletion that was intermediate between the 2 other studies [33]. Their findings were consistent with those of Miller et al. [30]. Andrew Holmes and his group [34] reported a more limited regional distribution of NR2B gene deletion than the other 2 studies. The apparent discrepancy between the NR2B CKO studies might be attributed to the extent of gene deletion in different areas of the brain.

In 2009, Lee et al. [35] demonstrated that the glycolytic enzyme glyceraldehyde 3-phosphate dehydrogenase (GAPDH) regulates mTOR activity by sequestering the Ras 
homolog enriched in brain (Rheb) [35]. Rheb is a small GTPase which functions as an upstream activator of mTOR. The investigators demonstrated that Rheb physically binds GAPDH, an interaction that seems to be regulated by glucose levels. When using culture media that contained $5 \mathrm{mM}$ glucose, the binding between Rheb and GAPDH was enhanced relative to the $25 \mathrm{mM}$ glucose condition. The interaction between Rheb and GAPDH correlated with inhibition of mTOR. Conversely, using a peptide derived from the binding domain of Rheb to GAPDH, the interaction between the 2 proteins was inhibited and mTOR was activated. These findings imply that agents blocking Rheb-GAPDH binding will activate mTOR and display antidepressant actions. GAPDH's role in glycolysis is to phosphorylate its substrate, glyceraldehye 3phosphate (G3P) to yield 1,3-bisphosphoglycerate. Interestingly, G3P, dose dependently disrupted the interaction between Rheb and GAPDH [35]. Thus, the glycolytic intermediate G3P seems to activate mTOR through inhibiting the Rheb-GAPDH interaction.

Previous work from our laboratory demonstrated that neuronal nitric oxide synthase (nNOS) nitrosylates GAPDH downstream of the NMDA receptor. In this context, GAPDH is nitrosylated on its catalytic cysteine $(\mathrm{C} 150)$ and loses its enzymatic activity as a consequence. Nitrosylated GAPDH binds to the E3 ubiquitin ligase seven in absentia homolog 1 (SIAH1). Excitotoxic levels of NMDA induced GAPDH-SIAH1 binding and translocation of both proteins to the nucleus to mediate NMDA-induced neural cell death [36]. The monoamine oxidase B inhibitor deprenyl blocked GAPDH nitrosylation, prevented its binding to SIAH1 and inhibited GAPDH translocation to the nucleus. Similar effects were observed using the deprenyl derivative, CGP3466B, that lacks the monoamine oxidase B inhibitory activity. Both compounds exerted these effects with high potency (subnanomolar levels) [37]. Notably, Kragten et al. [38] identified GAPDH as a specific target for CGP3466B.

We wondered whether GAPDH nitrosylation affects its binding to Rheb. In primary cortical cultures, nitric oxide (NO) donors, as well as NMDA, induced binding between GAPDH and Rheb. The NMDA-induced GAPDH-Rheb binding was blocked by NOS inhibitors and by preventing GAPDH nitrosylation using CGP3466B. Ketamine, however, inhibited GAPDH-Rheb binding. In HEK 293 cells, the catalytic cysteine mutant $C 150$ S GAPDH displayed reduced binding to Rheb. Overexpression of wild type but not C150S GAPDH resulted in reduction of Rheb expression. Conversely, GAPDH knockdown led to increased expression of Rheb [39]. These findings suggested a role for GAPDH nitrosylation in GAPDH-Rheb binding and in the regulation of Rheb expression. Interestingly, low levels of NMDA decreased Rheb protein levels within 15 min in primary cortical cultures derived from wild type and inducible NOS knockout but not from nNOS knockout mice. However, ketamine and the NR2B selective inhibitor CERC301 rapidly induced Rheb protein levels in primary cortical cultures. Moreover, ketamine increased Rheb protein levels in the mouse hippocampus within $30 \mathrm{~min}$ of intraperitoneal injection [39]. We reasoned that such rapid regulation of Rheb levels might be mediated at the level of protein degradation. Treating primary cortical cultures with the proteasome inhibitor brotezomib increased both Rheb protein levels and mTOR activation within $15 \mathrm{~min}$. Our data further demonstrate that NMDA-induced Rheb degradation is rescued by inhibiting the proteasome in primary cortical cultures. We identified an NMDA-induced ternary protein complex comprised of GAPDH, Rheb, and SIAH1. Purified Rheb physically binds SIAH1, and GAPDH nitrosylation enhances the formation of this ternary protein complex. Knocking down SIAH1 results in increased Rheb protein levels in primary cortical cultures [39]. Hence, SIAH1 presumably ubiquitylates Rheb leading to its degradation by the proteasome. However, there is no published study showing direct ubiquitylation of Rheb by SIAH1. Based on these findings, we argued that inhibition of GAPDH nitrosylation would result in an mTOR-dependent antidepressant effect. Using the

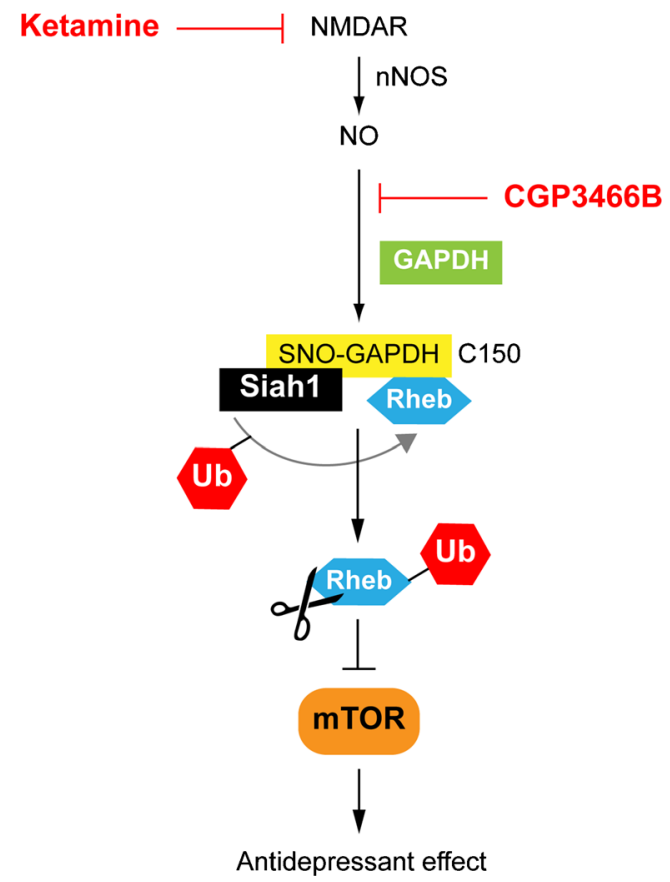

Fig. 1 A model of N-methyl-D-aspartate (NMDA) receptor (NMDAR) signaling to mechanistic target of rapamycin (mTOR). NMDA receptor signaling activates neuronal nitric oxide synthase (nNOS), which, in turn, nitrosylates glyceraldehyde 3-phosphate dehydrogenase (GAPDH) on its catalytic cysteine (C150). Nitrosylated GAPDH forms a ternary protein complex with seven in absentia homolog 1 (SIAH1) and the small GTPase Ras homolog enriched in brain (Rheb). SIAH1 ubiquitylates (Ub) Rheb, which is degraded by the proteasome. Degradation of Rheb leads to decreased mTOR activity. Ketamine's immediate antidepressant effect is presumably mediated by inhibiting this molecular cascade leading to mTOR activation. The GAPDH nitrosylation inhibitor CGP3466B blocks this cascade downstream of the NMDAR and results in an antidepressant-like effect in mice. $\mathrm{NO}=$ nitric oxide 
forced swimming test we demonstrated that inhibition of GAPDH nitrosylation by CGP3466B reduced the immobility time of mice, an effect that was reversed by the mTOR inhibitor rapamycin. Using the novelty suppressed feeding test, which differentiates actions of rapid versus traditional antidepressants, we demonstrated that CGP3466B elicited a rapid antdepressant-like effect that was reversed by rapamycin [39]. Our findings show that the NMDA receptor negatively regulates mTOR through NO-GAPDH-SIAH1-mediated degradation of Rheb. Ketamine blocks this negative regulation leading to stabilization of Rheb and allowing mTOR activation. The NMDA pathway might interface with AMPAR, extracellular signal-regulated kinase $1 / 2$, and phosphatidylinositide 3kinase signaling to activate mTOR in parallel with disinhibition through the NO-GAPDH-SIAH1-Rheb cascade. The nature of the circuit involvement that mediates the NOGAPDH-SIAH1-Rheb cascade is unclear in terms of excitatory and inhibitory neuronal balance.

Taken together, our data demonstrate that the NMDA receptor signals to mTOR through nNOS-mediated formation of a ternary protein complex containing SIAH1, Rheb, and GAPDH. SIAH1 ubiquitylates Rheb which is degraded by the proteasome. This, in turn, leads to mTOR inhibition. Blocking GAPDH nitrosylation using CGP3466B inhibits this cascade and results in an antidepressant-like effect in mice (Fig. 1) [39]. Our findings provide a molecular mechanism by which ketamine exerts its rapid antidepressant effect. It will be interesting to investigate whether the NO-GAPDHSIAH1-Rheb molecular cascade is involved in the sustained anti-depressant effect of ketamine.

Acknowledgments We thank Susan McTeer for organizing the manuscript. This work was supported by USPHS grant DA000266. The authors declare no conflicts of interest.

\section{References}

1. Javitt DC. Glutamate as a therapeutic target in psychiatric disorders. Mol. Psychiatry 2004; 9 (11): 984-997.

2. Anis NA, Berry SC, Burton NR, Lodge D. The dissociative anaesthetics, ketamine and phencyclidine, selectively reduce excitation of central mammalian neurones by $\mathrm{N}$-methyl-aspartate. $\mathrm{Br} J$ Pharmacol. 1983; 79 (2): 565-575.

3. Schwarcz R, Meldrum B. Excitatory aminoacid antagonists provide a therapeutic approach to neurological disorders. Lancet 1985; 2 (8447): 140-143.

4. Coyle JT, Tsai G, Goff DC. Ionotropic glutamate receptors as therapeutic targets in schizophrenia. Curr Drug Targets CNS Neurol Disord. 2002; 1 (2): 183-189.

5. Krystal JH, Karper LP, Seibyl JP, et al. Subanesthetic effects of the noncompetitive NMDA antagonist, ketamine, in humans. Psychotomimetic, perceptual, cognitive, and neuroendocrine responses. Arch Gen Psychiatry 1994; 51 (3): 199-214.

6. Berman RM, Cappiello A, Anand A, et al. Antidepressant effects of ketamine in depressed patients. Biol Psychiatry 2000; 47 (4): 351354.
7. Lally N, Nugent AC, Luckenbaugh DA, Ameli R, Roiser JP, Zarate CA. Anti-anhedonic effect of ketamine and its neural correlates in treatment-resistant bipolar depression. Transl Psychiatry 2014; 4: e469.

8. DiazGranados N, Ibrahim LA, Brutsche NE, et al. Rapid resolution of suicidal ideation after a single infusion of an N-methyl-Daspartate antagonist in patients with treatment-resistant major depressive disorder. J Clin Psychiatry 2010; 71 (12): 1605-1611.

9. Zarate CA Jr, Singh JB, Carlson PJ, et al. A randomized trial of an $\mathrm{N}$-methyl-D-aspartate antagonist in treatment-resistant major depression. Arch Gen Psychiatry 2006; 63 (8): 856-864.

10. Li N, Lee B, Liu RJ, et al. mTOR-dependent synapse formation underlies the rapid antidepressant effects of NMDA antagonists. Science 2010; 329 (5994): 959-964.

11. Li N, Liu RJ, Dwyer JM, et al. Glutamate N-methyl-D-aspartate receptor antagonists rapidly reverse behavioral and synaptic deficits caused by chronic stress exposure. Biol. Psychiatry 2011; 69 (8): 754-761.

12. Zanos P, Moaddel R, Morris PJ, et al. NMDAR inhibitionindependent antidepressant actions of ketamine metabolites. Nature 2016; 533 (7604): 481-486.

13. Trullas R, Skolnick P. Functional antagonists at the NMDA receptor complex exhibit antidepressant actions. Eur J Pharmacol. 1990; 185 (1): 1-10.

14. Preskorn SH, Baker B, Kolluri S, Menniti FS, Krams M, Landen JW. An innovative design to establish proof of concept of the antidepressant effects of the NR2B subunit selective N-methyl-Daspartate antagonist, CP-101,606, in patients with treatmentrefractory major depressive disorder. J Clin Psychopharmacol. 2008; 28 (6): 631-637.

15. Ferguson JM, Shingleton RN. An open-label, flexible-dose study of memantine in major depressive disorder. Clin Neuropharmacol 2007; 30 (3): 136-144.

16. Lenze EJ, Skidmore ER, Begley AE, Newcomer JW, Butters MA, Whyte EM. Memantine for late-life depression and apathy after a disabling medical event: a 12-week, double-blind placebo-controlled pilot study. Int J Geriatr Psychiatry 2012; 27 (9): 974-980.

17. Zarate CA, Singh JB, Quiroz JA, et al. A double-blind, placebocontrolled study of memantine in the treatment of major depression. Am J Psychiatry 2006; 163 (1): 153-155.

18. Lord B, Wintmolders C, Langlois X, Nguyen L, Lovenberg T, Bonaventure P. Comparison of the ex vivo receptor occupancy profile of ketamine to several NMDA receptor antagonists in mouse hippocampus. Eur J Pharmaco. 2013; 715 (1-3): 21-25.

19. Muhonen LH, Lönnqvist J, Juva K, Alho H. Double-blind, randomized comparison of memantine and escitalopram for the treatment of major depressive disorder comorbid with alcohol dependence. $J$ Clin Psychiatry 2008; 69 (3): 392-399.

20. Murrough JW, Iosifescu DV, Chang LC, et al. Antidepressant efficacy of ketamine in treatment-resistant major depression: a two-site randomized controlled trial. Am J Psychiatry 2013; 170 (10): 1134 1142.

21. Gideons ES, Kavalali ET, Monteggia LM. Mechanisms underlying differential effectiveness of memantine and ketamine in rapid antidepressant responses. Proc Natl Acad Sci U S A 2014; 111 (23): 8649-8654.

22. Johnson JW, Glasgow NG, Povysheva NV. Recent insights into the mode of action of memantine and ketamine. Curr Opin Pharmacol. 2015; 20: 54-63.

23. Adachi N, Numakawa T, Kumamaru E, et al. Phencyclidineinduced decrease of synaptic connectivity via inhibition of BDNF secretion in cultured cortical neurons. Cereb Cortex 2013; 23 (4): 847-858.

24. $\mathrm{Xu}$ J, Kurup P, Baguley TD, et al. Inhibition of the tyrosine phosphatase STEP61 restores BDNF expression and reverses motor and 
cognitive deficits in phencyclidine-treated mice. Cell Mol Life Sci 2016; 73 (7): 1503-1514.

25. Garcia LS, Comim CM, Valvassori SS, et al. Acute administration of ketamine induces antidepressant-like effects in the forced swimming test and increases BDNF levels in the rat hippocampus. Prog Neuropsychopharmacol Biol Psychiatry 2008; 32 (1): 140-144.

26. Autry AE, Adachi M, Nosyreva E, et al. NMDA receptor blockade at rest triggers rapid behavioural antidepressant responses. Nature 2011; 475 (7354): 91-95.

27. Nosyreva E, Autry AE, Kavalali ET, Monteggia LM. Age dependence of the rapid antidepressant and synaptic effects of acute NMDA receptor blockade. Front Mol Neurosci 2014; 7: 94.

28. Maeng S, Zarate CA, Du J, et al. Cellular mechanisms underlying the antidepressant effects of ketamine: role of alpha-amino-3-hydroxy-5-methylisoxazole-4-propionic acid receptors. Biol Psychiatry 2008; 63 (4): 349-352.

29. Paul RK, Singh NS, Khadeer M, et al. (R,S)-Ketamine metabolites $(\mathrm{R}, \mathrm{S})$-norketamine and $(2 \mathrm{~S}, 6 \mathrm{~S})$-hydroxynorketamine increase the mammalian target of rapamycin function. Anesthesiology 2014; 121 (1): 149-159.

30. Miller OH, Yang L, Wang CC, et al. GluN2B-containing NMDA receptors regulate depression-like behavior and are critical for the rapid antidepressant actions of ketamine. Elife 2014; 3: e03581.

31. Brigman JL, Wright T, Talani G, et al. Loss of GluN2B-containing NMDA receptors in CA1 hippocampus and cortex impairs longterm depression, reduces dendritic spine density, and disrupts learning. J Neurosci 2010; 30 (13): 4590-4600.
32. Goebbels S, Bormuth I, Bode U, Hermanson O, Schwab MH, Nave KA. Genetic targeting of principal neurons in neocortex and hippocampus of NEX-Cre mice. Genesis 2006; 44 (12): 611-621.

33. Engelhardt von J, Doganci B, Jensen V, et al. Contribution of hippocampal and extra-hippocampal NR2B-containing NMDA receptors to performance on spatial learning tasks. Neuron 2008; 60 (5): 846-860.

34. Kiselycznyk C, Svenningsson P, Delpire E, Holmes A. Genetic, pharmacological and lesion analyses reveal a selective role for corticohippocampal GLUN2B in a novel repeated swim stress paradigm. Neuroscience 2011; 193: 259-268.

35. Lee MN, Ha SH, Kim J, et al. Glycolytic flux signals to mTOR through glyceraldehyde-3-phosphate dehydrogenase-mediated regulation of Rheb. Mol. Cell. Biol. 2009; 29 (14): 3991-4001.

36. Hara MR, Agrawal N, Kim SF, et al. S-nitrosylated GAPDH initiates apoptotic cell death by nuclear translocation following Siah1 binding. Nat Cell Biol 2005; 7 (7): 665-674.

37. Hara MR, Thomas B, Cascio MB, et al. Neuroprotection by pharmacologic blockade of the GAPDH death cascade. Proc Natl Acad Sci U S A 2006; 103 (10): 3887-3889.

38. Kragten E, Lalande I, Zimmermann K, et al. Glyceraldehyde-3phosphate dehydrogenase, the putative target of the antiapoptotic compounds CGP 3466 and R-(-)-deprenyl. J Biol Chem 1998; 273 (10): 5821-5828.

39. Harraz MM, Tyagi R, Cortes P, Snyder SH. Antidepressant action of ketamine via mTOR is mediated by inhibition of nitrergic Rheb degradation. Mol Psychiatry 2016; 21 (3): 313-319. 